\title{
La naturaleza, fuente de vida y diversidad que se debe conservar. El discurso de Juan sobre la naturaleza en la clase de Ciencias
}

THE NATURE, SOURCE OF LIFE AND DIVERSITY TO BE CONSERVED. JOHN'S SPEECH ABOUT NATURE IN SCIENCE CLASS

A NATUREZA, FONTE DE VIDA E DIVERSIDADE QUE SE DEVE CONSERVAR. O DISCURSO DE JUAN SOBRE A NATUREZA NA CLASSE DE CIÊNCIAS

Andrés Arturo Venegas Segura* / andresvs5@yahoo.com, aavenegas@udistrital.edu.co

\section{Resumen}

Se presenta el discurso de Juan sobre la naturaleza resaltando los valores éticos, estéticos, emocionales, regulatorios, enmarcados por un ethos de la etnia Sikuani, donde los conocimientos escolares son retomados con base en su cosmovisión. La interpretación es un resultado de la Tesis Doctoral "Ideas de naturaleza de niños y niñas Sikuani y Llaneros de la clase de Ciencias del cuarto grado de Básica Primaria del Colegio Agropecuario Silvino Caro Heredia, en el Corregimiento del Viento, Vichada". La metodología y la interpretación sefundamentan en los "conglomerados de relevancias" y la triangulación de instrumentos.

\section{Summary}

We present a discourse on the nature of Juan highlights its ethical, aesthetic, emotional, regulatory, framed by an ethos Sikuani where school knowledge are taken up based on their worldview. As a result the interpretation of the doctoral thesis "Ideas de naturaleza de niños y niñas Sikuani y Llaneros de la clase de Ciencias del cuarto grado de Básica Primaria del Colegio Agropecuario Silvino Caro Heredia, en el Corregimiento del Viento, Vichada". The methodology and interpretation are based on Conglomerados de Relevancias and triangulation of instruments.

\section{Resumo}

Nósapresentamos um discurso sobre a natureza da Juan destacando sua ética, estética, emocional, regulamentar, emoldurado por umethos Sikuani onde o conhecimento da escolasão tomadas com base emsuavisão de mundo. Como resultado, a interpretação das teses de doutorado "Ideas de naturaleza de niños y niñas Sikuani y Llaneros de la clase de Ciencias del cuarto grado de Básica Primaria del Colegio Agropecuario Silvino Caro Heredia, en el Corregimiento del Viento, Vichada”. A metodologia e interpretação são baseadas em Conglomerados de Relevancias e triangulação de instrumentos.

\footnotetext{
* Universidad Distrital Francisco José de Cladas Doctorado interinstitucional en educación INTERCITEC. Doctor en Educación de la Universidad Distrital Francisco José de Caldas. Especialización en Bioingeniería de la Universidad Distrital. Licenciado en Física de la Universidad Distrital y Antropólogo de la Universidad Nacional de Colombia.
}

Fecha de recepción: 14 de septiembre de 2012 / Fecha de aprobación: 18 de octubre de 2012 
Palabras clave

Idea de naturaleza, madre naturaleza, Sikuani, Vichada.

\section{Keywords}

Idea of nature, mother nature, Sikuani, Vichada.

\section{Palavras-chave}

Idéia de natureza, mãe natureza, Sikuani, Vichada.

\section{Introducción}

Considerando la emergencia y la configuración de perspectivas sobre el mundo natural en un escenario escolar, específicamente en la clase de ciencias naturales, se pretende evidenciar las ideas y los criterios de valor que las orientan, para que el proceso se útil como motor de reconocimiento del otro, de su universo interpretativo, y a la vez como elemento de la reflexión pedagógica y didáctica en escenarios escolares diversos.

En consideración a ello, el presente artículo tiene como objeto dar a conocer la interpretación del discurso de Juan sobre la naturaleza, basado en los "conglomerados de relevancias" (Molina, 2008, 2007a, 2007b, 2004, 2002, 2000), el cual gira en torno a los diálogos con él, (en una entrevista semi-estructurada), en un escrito, Carta al extraterrestre, y un dibujo, Dibujo sobre la naturaleza, los cuales se constituyen en los instrumentos de recolección de información.

Además, se introducen comentarios que son parte del diario de campo, para enriquecer la interpretación. El contexto en el cual se llevó a cabo la investigación fue la clase de Ciencias del cuarto grado del Colegio Silvino Caro Heredia, en el Departamento del Vichada, escenario con alta diversidad cultural en el que se encuentran niños y niñas con ascendencia Sikuani, Piapoco, Llaneros y Colonos.

Por lo anterior, se asume la hipótesis de que se trata de una clase de ciencias en la cual coexisten valores y creencias culturalmente diferenciados, lo cual atiende a negociaciones y traspasos de fronteras culturales como las tratadas por diversos autores, entre ellos: Aikenhead, 2001a, 2001b, 1997, 1996; Aikenhead y Jegede, 1999; Aikenhead y Olugbemiro, 1999; Candau, 2006; Cobern y Loving 2001; Cobern, 1996a, 1996b, 1994, 1991; Jegede y Ogawa, 1999; Jegede, 1995; Jegede y Okebukola, 1991; June, 1999; Kawasaki, 1997, 1996; Molina, 2008, 2007a, 2007b, 2004, 2002, 2000; Molina y Mojica, 2005, 2004; Ogawa, 2000, 1995, 1989, 1986; Okhee, 2003, 1999; Riggs 2004; Shu-Chiu, 2005).

Así, las ideas, pensamientos y afirmaciones, expresadas por los niños y niñas son negociadas de formas diversas con su cultura en un contexto escolar. Por lo anterior, el Discurso de Juan da cuenta de dichos intercambios. En su idea de naturaleza se hacen presentes sus conocimientos tradicionales Indígenas, Llaneros

Rollos nacionales / La naturaleza, fuente de vida y diversidad 
y escolares. En consideración a ello, se determinó el "Conglomerado de Relevancias" en la idea de naturaleza de Juan, por medio de su discurso, encontrándose los criterios: Espiritual, de Utilidad, Naturalista, ÉticoEstético-Emocional (E-E-E) y Espacial; así mismo, las jerarquías que orientan estos criterios.

El texto está compuesto por cuatro apartados que corresponden a: 1) Introducción. 2) Referente conceptual y metodología. 3) Resultados e Interpretación, dividido en, a) Presentación de Juan en su contexto. b) La descripción general de las jerarquías del Conglomerado de Relevancias. c) Tipos de criterios y jerarquías asociadas al discurso de Juan; 4) Se presenta unas consideraciones finales a manera de conclusiones.

\section{Referente conceptual y metodología}

La metodología de la presente investigación se enmarca dentro de un enfoque cualitativo que permite aproximarse a una interpretación cultural del universo interpretativo del otro. En este sentido, no se utilizan categorías predefinidas, sino que por el contrario, se trata de categorías abiertas. Se parte de un marco interpretativo de las ideas y de los diálogos a estudiar por medio de los Conglomerados de Relevancias.

Molina $(2000,2004)$ propone el concepto de Conglomerado de Relevancias, que estudia las interrelaciones entre conocimiento y cultura, postulando que el significado es dependiente del contexto y que la significación de la experiencia se puede llevar al lenguaje, en el cual se evidencian: "valores, conglomerados de relevancias que se expresan, y señalan la creencia, legitimidad, convivencia, de aquello que es significativo y que ellos [los conglomerados] remiten a los contextos culturales" (Molina, 2004, p. 193).

Además, el Conglomerado de Relevancias se propone con el fin de caracterizar las visiones y perspectivas sobre el mundo natural, resultantes de los intercambios culturales que se presentan en una sociedad caracterizada por su diversidad cultural. La propuesta se basa en Ricoeur y la idea de valor como decisión, que permite resolver las opciones de significar presentes en los intercambios entre culturas (Molina, 2000, 2004).

En el mismo orden, esta categoría se construyó bajo supuestos, como: los niños y niñas elaboran significados; los significados intermedian el proceso de conocer; los procesos de significación se constituyen junto con criterios de selección, en la construcción del conocimiento, ya que no todo lo que se ve es percibido, no todo lo que sucede es valorizado con el mismo peso; los significados se expresan en el lenguaje; la cultura determina los esquemas de significación transmitidos históricamente, que pueden ser reconstruidos a partir de sus representaciones, elaboraciones simbólicas e interrelaciones, así como a partir de sus valores, actitudes y demás manifestaciones; y los significados pueden ser estudiados por la semántica (Molina 2008).

En consonancia, las categorías metodológicas adoptadas se articulan epistemológicamente con el concepto de Conglomerado de Relevancias (Molina, 2000, 2008) y son tres: a) Idea de narrativa, esto es, la forma como se concebirán las cartas de los niños y las niñas, sus diálogos y dibujos; b) Contenido semántico, los criterios de interpretación de las declaraciones de los niños y las niñas y c) Contexto cultural. Con referencia a este marco se procedió a valorar la voz del otro; cada aspecto de su discurso es visualizado como un espacio de significado, encontrándose una serie de criterios de valor que dan la posibilidad de apreciar positivamente todos los lugares que narran, quieren expresar o mostrar en su discurso sobre la naturaleza.

El discurso en torno a la idea naturaleza está implicado en las narraciones, dibujos comentados y diálogos. Las cartas proporcionan una descripción de las ideas que el niño tiene de la naturaleza, al encontrarse con un ser ajeno al planeta tierra, generando el proceso de extrañamiento, donde se busca que la descripción sea lo más rica posible.

Por su parte, el dibujo facilita el diálogo sobre la naturaleza, ya que por medio de este recurso el niño puede expresar otras preocupaciones, ideas, valores; así mismo, las imágenes informan sobre sus ideas, las historias que quiere contar, lo relevante y cómo percibe ciertos espacios (Moura De Macedo, 2007); mientras que en el dialogo es posible profundizar en su discurso, sus afirmaciones son contextualizadas en su contexto cultural y escolar. Estas tres formas de acceder a su idea de naturaleza permiten que Juan exprese sus ideas en tres contextos de uso diferenciados, encontrándose sus criterios de valor, Conglomerado de Relevancias, en torno de la idea de naturaleza.

Las interpretaciones de su idea sobre la naturaleza se realizan a partir de los instrumentos mencionados y de su relevancia documental; esta última, basada en la metodología de análisis de documentos, los textos utilizados se clasifican, analizan y se explican con la idea de comprender el contexto cultural de Juan y sus afirmaciones. Los documentos fueron fichados con base en los argumentos dados por los diferentes autores para 
describir los contextos culturales de las afirmaciones de Juan, lo cual es útil para referir las diversas ideas que expone, permitiendo el acercamiento y alejamiento de planteamientos sobre la naturaleza.

\section{Resultado e interpretación}

\section{a. Juan}

Juan es un niño de 10 años que nació en la Orinoquia colombiana, su tiempo lo invierte estudiando, jugando, pescando, cazando y aprendiendo con sus parientes Sikuani y Llaneros. Encuentra en el canto una forma de manifestar sus sentimientos y expresarse ante la vida, profesando gran amor y pasión por la naturaleza. La Orinoquia es fundamental para él y le da felicidad, le permite el desarrollo de procesos de identidad y pertenencia hacia su región; al hablar de ella se siente en disposición para enseñar y dejar un mensaje de cuidado hacia ese maravilloso mundo natural que describe. Por otro lado, sus maestros dicen que es un niño muy feliz, buen estudiante, siempre atento a las explicaciones.

\section{b. Descripción General de las jerarquías del Conglo- merado de Relevancias}

La interpretación de las respuestas de los tres instrumentos trabajados con Juan, permite observar las jerarquías que conforman el Conglomerado de Relevancias de su idea de naturaleza. Esta se configura a partir del criterio espiritual, naturalista, de utilidad, espacial y Estético-Ético-Emocional. Estos se encuentran relacionados en diferentes niveles, como se puede observar en el Gráfico 1.

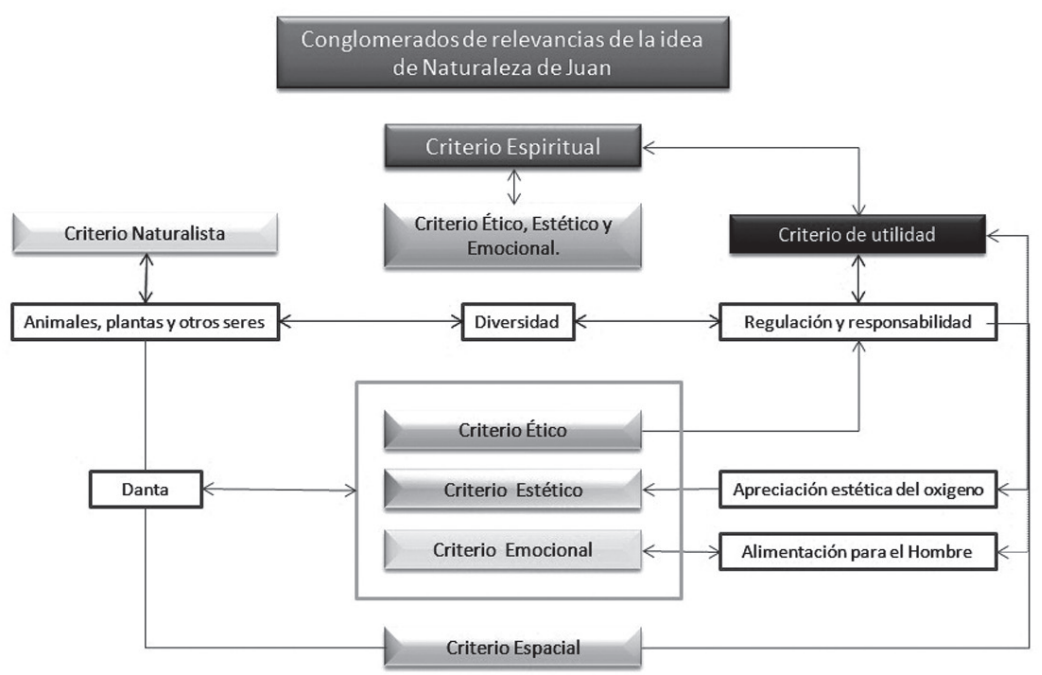

- Demuestra una Relación Horizontal - Demuestra una Relación de Dependencia
Las Jerarquías indicadas en el Conglomerado de Relevancias implican los criterios antes mencionados y sus relaciones se describen a continuación:

- Jerarquía de dependencia 1 (JD1): Configurada entre el criterio Espiritual con el E-E-E y de utilidad. Juan manifiesta una idea de naturaleza cargada de la noción de "Madre Naturaleza": para Juan la naturaleza tiene espíritu. El criterio E-E-E específicamente el estético se fundamenta en esta idea de espiritualidad: la naturaleza es bella e igualmente debe ser cuidada. Estos tres criterios, espiritual, estético y ético son generadores de emociones. Pese a que Juan no puede explicar el espíritu de la naturaleza, éste se encuentra presente en sus creencias y está fuertemente arraigado a ellas.

Por otra parte, su espiritualidad le lleva a una manifestación de supuestos éticos a la hora de hacer uso de la naturaleza; en este sentido, el criterio espiritual regula también el criterio de utilidad, vinculando el criterio E-E-E, específicamente el ético. De esta forma Juan busca actuar bien frente a la naturaleza, o sea no devastar, no utilizar más de lo debido. Así se justifica la dependencia entre los criterios E-E-E y de utilidad con el Espiritual, donde los dos primeros se constituyen como vía de expresión del tercero.

- Jerarquía de dependencia 2 (JD2): Configurada a partir del criterio E-E-E, específicamente el nivel estético y los criterios de utilidad y naturalista. El criterio estético entabla una relación de dependencia que hace énfasis en la belleza e importancia del oxígeno para la vida. En este caso, la utilidad del oxígeno se refiere a su importancia para la vida, aspecto que Juan infiere de lo enseñado en la clase de ciencias; así concluye que al ser el oxígeno útil para la vida, es bello.

Gráfico 1. Jerarquías en el Conglomerado de Relevancias para Juan 
Por otra parte, el criterio naturalista se origina en el conocimiento escolar con respecto al oxígeno, hecho que no es resultado de su experiencia empírica con la naturaleza. Se plantea también la relación entre el criterio de utilidad y el criterio E-E-E, específicamente en el nivel emocional. La utilidad de la naturaleza está referida ahora al consumo de frutas, hecho que considera importante para su alimentación, y que le produce además gusto y agrado, evidenciando de esta forma el criterio emocional.

- Jerarquía de dependencia 3 (JD3): Configurada a partir de los criterios E-E-E, específicamente el nivel ético, con los criterios espacial y de utilidad. Se encontró que el criterio de utilidad siempre se relaciona con el criterio ético y espacial, pues la utilidad que prestan los animales, plantas y otros seres a los humanos, está regulada por una manera correcta de actuar. El espacio y los seres de la naturaleza solo tienen un nivel limitado de uso por parte del ser humano, lo cual significa también que la naturaleza es objeto de aprovechamiento colectivo y no individual.

- Jerarquía Horizontal (JH): Configurada entre los criterios naturalista y de utilidad. El conocimiento de la naturaleza permite su utilización, configurándose sus ideas de oxígeno, territorio, prácticas de pesca, cultivo y cacería, diversidad de animales y plantas, descripción de pautas de comportamiento y alimentación.

\section{c. Tipos de Criterios y jerarquías asociadas en el Discurso de Juan}

En este apartado se analizan los criterios encontrados en el discurso de Juan, ofreciendo una descripción detallada de cada uno, y un análisis basado en sus narraciones, con el fin de mostrar cómo se estructuran. Igualmente, se presenta la forma en que se establecieron las jerarquías descritas en el apartado anterior.

\section{Criterio Naturalista y jerarquías asociadas}

En los diálogos con Juan se encuentran dos aspectos relevantes en cuanto a la naturaleza y uso de su criterio naturalista; el primero ligado a la diversidad de animales y plantas, en el cual se distinguen distintas formas de agrupamientos y conocimientos empíricos de los organismos. El segundo es una relación directa entre el lugar donde habitan los animales, sus pautas de comportamiento y alimentación; esto aparece como caso puntual en sus dibujos, la carta y la entrevista a la Danta, mostrando la importancia de este animal. Lo anterior está ligado al conocimiento de su territorio.

El criterio naturalista, está presente cuando considera la diversidad de plantas y animales como un elemento fundamental para describir la Naturaleza: "En la naturaleza hay muchos animales y plantas" (Carta), y cuando se refiere a los animales que hay en los Llanos Orientales, "la Danta, el Chigüiro, el Cajuche, el Pavo Real, el Saino, el Venao, el Purre" (Carta).

Un aspecto que hace posible un contraste está dado por el dominio de tres tipos de agrupamientos: el primero se refiere a todos los seres que corresponden a una misma denominación, por ejemplo, los que Ilama "Cajuche", "Saino", "Purre", "Venao"; el segundo, a todos los seres que son animales, plantas e insectos; y el tercero, a todos los seres vivos. En este sentido, según Juan: “La naturaleza es amplia y llena de seres vivos como animales, plantas e insectos". Los dos primeros grupos se refieren a sus experiencias empíricas y el tercero a una agrupación fruto de su conocimiento escolar.

Juan también expone el criterio naturalista como algo ligado a la diversidad, ahora referido a las serpientes: "Cuatro Narices, Cascabel, Macabrel, Pudridora, Cazadora, Loro, Guio" (Entrevista), este conocimiento está asociado a la relación entre éstas y el ser humano y resalta la peligrosidad de estos animales por los venenos que pueden inyectar y las laceraciones que logran ocasionar. Así mismo, comenta sobre los remedios que utilizan en la zona para calmar los síntomas de la mordedura de algunas de estas serpientes: "el Chimú [...] Se lo comen y se echan donde le mordió y [...] medio le calma el dolor" (Entrevista). El conocimiento sobre el efecto del "Chimú" hace parte del contexto cultural de Juan y se fundamenta en su experiencia.

Otro nivel en el uso de palabras, ya no referido a agrupaciones y descripciones de seres, es la emergencia del término oxígeno y otros asociados, como respiración e implícitamente fotosíntesis; así, el criterio naturalista es orientado por el conocimiento escolar. El oxígeno permite la respiración a los animales y la vida a las plantas, de tal forma, Juan considera que el oxígeno tiene un vínculo cercano con la producción de alimento para las plantas y los animales, pero implícitamente relaciona que la propia producción de alimentos por 
parte de las plantas, da como resultado la generación de oxígeno, que claramente está asociado a la respiración de las plantas y animales y a la posibilidad de realizar las actividades vitales: "los animales necesitan alimentarse para vivir, las plantas producen su propio alimento pero para alimentarse necesitan oxígeno para poder respirar" (Carta). En este caso, JD2 se apoya en los conocimientos empíricos valorados como hechos importantes en su contexto cultural y los conocimientos escolares; así, el criterio naturalista en esta jerarquía tiene una doble cara.

Nuevamente se considera el hecho de que las plantas están al servicio de los animales, proveyéndoles de oxígeno y alimento; Juan, de nuevo, retoma este conocimiento dentro de sus argumentos sobre qué es la naturaleza: "las plantas brindan el oxígeno a los animales y sin el oxígeno los animales no podrían vivir" (Entrevista), ya que sin las plantas los animales no se pueden alimentar. Sin embargo, tanto las plantas como los animales son igualmente importantes desde una perspectiva Holística, al considerar dinámicas más integrales, "sin las plantas tampoco podrían comer los animales, no encontrarían plantas, nada de eso para ellos alimentarse" (Entrevista).

\section{Criterio de Utilidad y jerarquías asociadas}

El criterio de utilidad está relacionado con los criterios naturalista, E-E-E y espacial, conformando diferentes jerarquías (JD2, JD3 y JH). Se hace evidente en el momento en que Juan explica actividades realizadas usualmente en la región, como la cacería, la pesca y la siembra, las cuales permiten la observación de diversas características de animales y vegetales; tales descripciones están acompañadas por apreciaciones y emociones.

Juan muestra algunas relaciones entre el ser humanos y la naturaleza que dan cuenta de su comprensión sobre la utilidad que los animales y las plantas prestan al ser humano. Comprensión precedida por una posición ética con referencia al uso indebido de los animales, que ocasiona la extinción de los mismos, "pero si [...] el hombre no los sabe utilizar se van agotar" (Carta). Revelando JD3 que considera una regulación de esta utilidad de la naturaleza, en el uso de los animales. Así el E-E-E, se constituye como elemento regulador del criterio utilidad.
La Danta adquiere un significado especial, ya que es el único animal que aparece en su dibujo (Ilustración 1) y le proporciona alimentación al ser humano, utilidad que está asociada al gusto por su carne: "Porque me gusta mucho la carne de la Danta" (Entrevista), seguido por una explicación de la forma cómo es preparada, lo cual le lleva a expresar emociones de alegría al referirse a este alimento: "Se puede preparar en frita o con sopa" (Entrevista). Constituyéndose JD3.

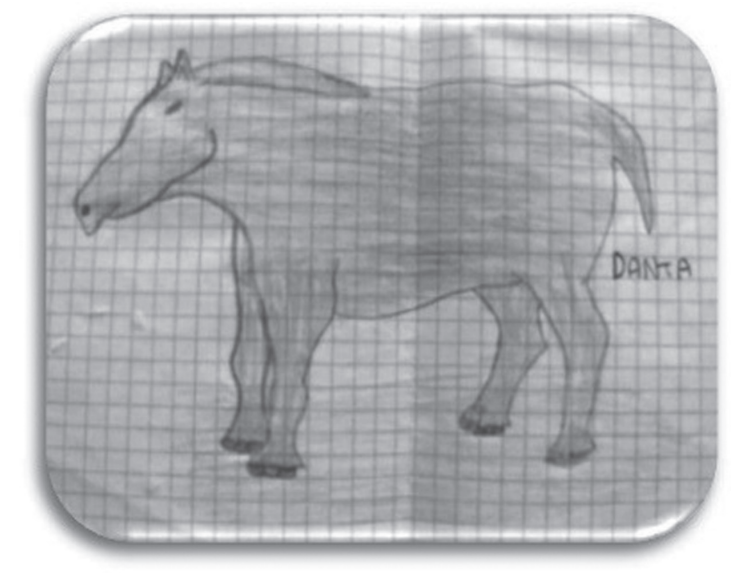

Ilustración 1. Dibujo sobre la naturaleza de Juan

La Danta está ligada al criterio de utilidad, no obstante, cuando Juan describe las pautas de comportamiento de este animal y su modo de obtener alimento, las articula con el criterio naturalista, narrando cómo los cultivos de yuca son parte de la dieta de este animal, "Comen cultivos de yuca" (Entrevista).

Por otra parte, Juan explica la problemática de la relación Danta-cultivos para ciertos pobladores, pues en diversas ocasiones la irrupción del animal en los sembradíos ocasiona su muerte, ya que los cultivos son fundamentales para la alimentación de los habitantes de esta zona: "Pero cuando se entran a los yuqueros hay veces las matan porque se comen los cultivos, las matan porque a las personas no les gustan" (Entrevista). Es necesario aclarar que por la forma en que Juan aborda este punto, la tonalidad de su voz, la afirmación "Ias matan porque a las personas no les gustan", como otros aspectos vividos en la entrevista, hace evidente el repudio que siente por la muerte del animal, configurándose el criterio E-E-E, cuando asocia lo ético (la regulación para no matar Dantas) y lo emocional, por la pérdida de la vida de este animal, estableciendo JD3. Juan menciona las diferentes clases de yuca, dando cuenta de las variedades de esta planta, es decir, la 
diversidad de la misma, hecho que articula el criterio naturalista al de utilidad, "hay una amarga, una amarga y otra dulce" (Entrevista). Constituyéndose JH ligada a procesos de producción de alimento. Las relaciones de estos cultivos con su entorno familiar hacen que se consolide un conocimiento tradicional en correspondencia directa con el criterio de utilidad y el criterio naturalista, $\mathrm{JH}$; pues implica el manejo y uso de diversos saberes frente a esta planta, "Investigador: ¿Y tú dónde conoces cultivos de yuca?- Juan: Donde mi padrino (...) por allá en una finca" (Entrevista).

Juan comenta sobre el consumo de frutas y presenta el papel de éstas en su alimentación, vislumbrándose un JH. Así mismo, emerge el criterio E-E-E, específicamente el emocional, resaltando una apreciación relevante, que constituye así un JD2; de este modo, cita en su entrevista al mango, la guayaba, el coco, el mamoncillo y el pendare, explicando que su consumo se debe a su agradable sabor: "Investigador: ¿Qué plantas dices que son importantes aquí en el Llano?- Juan: El mango [...] el coco, el pendare" (Entrevista). Al preguntar a Juan sobre qué fruta es el pendare, él aclara que su sabor se parece al chicle, para la comprensión del investigador: "Es una pepa como así verde y por dentro tienen como unas pepas de chicle, entonces uno se las come y son ricas" (Entrevista).

El criterio de utilidad se evidencia también cuando el niño se refiere al servicio que le prestan las plantas a los animales, concibiéndose un $\mathrm{JH}$, un ejemplo se da cuando comenta las diferencias en la alimentación de la Danta y el Venado, dando cuenta de su conocimiento sobre s interacción con las plantas e insectos quienes les proveen alimento: "Yo no sé cómo se llama esa frutica que bajan de los árboles y la comen las Dantas, y los venados casi no comen eso, comen es pastos y otros insectos o cosas que encuentran por ahí" (Entrevista).

Por otro lado, la pesca obedece al criterio de utilidad, y se constituye en una experiencia que permite a Juan establecer las relaciones alimenticias entre unos animales y otros, el caso de las sardinitas: "entonces uno coge la carnada, una sardinita y la mete así y la tira, y uno pone el nylon así, entonces está el agua y cuando levantó así es porque lo están Ilevando y uno hala así" (Entrevista), aspecto referido al criterio naturalista, constituyéndose un $\mathrm{JH}$; este saber le permite realizar la pesca de una manera efectiva. Además, la pesca tiene unas formas específicas de realizarse, unos utensilios específicos, como el nylon.
Juan relaciona esta actividad con el tipo de pez y sus experiencias de pesca: "los caribes son [...] duros de sacar -porque casi no he sacado- sí saco, pero lo que más he sacado es pabon y guabina y chuanos" (Entrevista). Ahora bien, se constituye un JH en esta práctica, ya que Juan integra las características propias de algunos peces, su diversidad, sus lugares de vida y las condiciones ambientales en las que habitan, "Los chuanos son como unos salomones (...) sino que tienen la cabeza lisa. Las guabinas son lisas" (Entrevista).

\section{Criterio Espacial y jerarquías asociadas}

Al mencionar que "La naturaleza es amplia", Juan se está refiriendo a una apreciación de la dimensión espacial, consolidando el criterio espacial ligado a los criterios de utilidad y naturalista. Además, es posible vislumbrar el criterio espacial cuando explica el lugar donde habita la Danta: "En el monte" (Entrevista), acepción de uso regional referida a tradiciones de las comunidades Ilaneras y que corresponde a un lugar en el cual no viven seres humanos. En el apartado anterior se consolida un JD3, cuando Juan nombra los conflictos entre algunos pobladores y este animal debidos a su irrupción en los cultivos de yuca, situación en la que Juan repudia la matanza de la Danta, hecho que esta por fuera de los límites de su regulación ética.

Más adelante, Juan argumenta la necesidad de una regulación sobre el manejo del espacio, pues la relación monte-siembra-Danta se torna paradójica, ya que la Danta vive en el monte y allí encuentra su alimento, pero también en el monte se desarrolla la actividad de la siembra, y gracias a la expansión de esta actividad la Danta ha perdido su espacio, su alimento y ha adoptado los cultivos, hecho que la conduce a la muerte. Así se interpreta que Juan, debido a sus conocimientos ancestrales da cuenta de una regulación del espacio tanto para el Hombre como para los demás animales, ya que considera cómo el monte suple las necesidades alimenticias de todos (animales, plantas y el Hombre), el monte es fuente de vida.

\section{Criterio Ético-Estético-Emocional y jerarquías asociadas}

El criterio $E-E-E$ es fundamental en las ideas sobre la naturaleza de Juan, dado que le permite valorar a la naturaleza como algo bello, que debe ser querido, respetado y cuidado, constituyendo así un JD1, ya que 


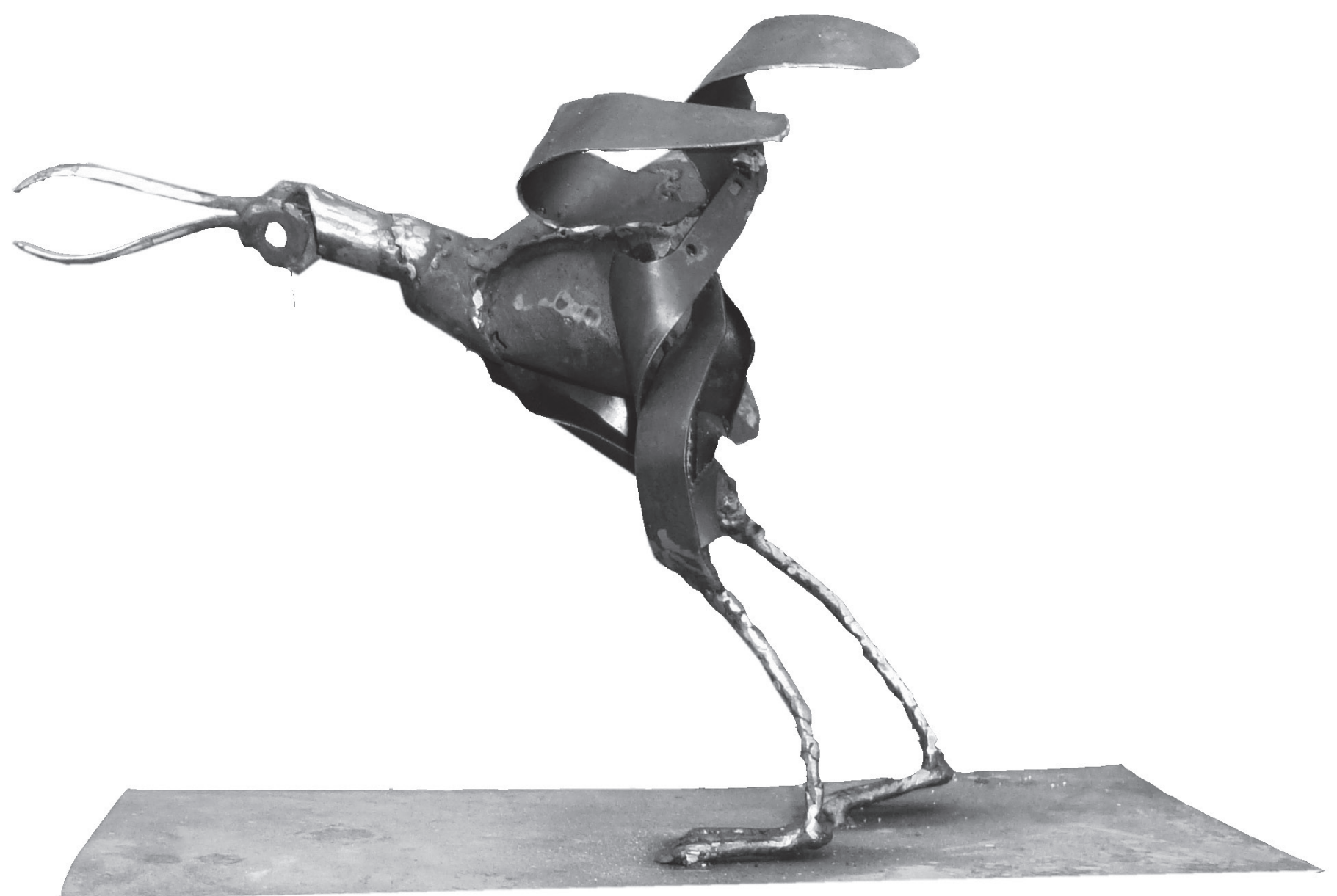

Titulo: Tingua

Dimensiones: $25 \times 35 \times 10 \mathrm{~cm}$

Tecnica: Escultura en metal

Año: 2011 
estos aspectos se articulan con el criterio espiritual referido a la naturaleza. Juan la considera bella: "la naturaleza es muy bella porque nos brinda oxigeno" (Carta), evidenciando el criterio estético ligado al criterio de utilidad; de esta manera, el oxígeno es bello pues es útil para el ser humano, constituyéndose un JD2, resaltando el papel fundamental del oxígeno para la vida, que se articula en sus ideas sobre la naturaleza y la vida, sin embargo, este concepto es inferido a partir de sus conocimientos escolares.

Así mismo, Juan se refiere a la naturaleza con expresiones que demuestran sentimientos de cercanía hacia ella (el querer): "lo que yo más quiero de lo que hay en el mundo es la naturaleza" (Carta). Resalta sentimientos acerca del bienestar que le brinda la naturaleza, en especial el alimenticio. Además, existe una idea de respeto y regulación hacia la naturaleza, que se traduce en el criterio ético para el manejo de los animales, "si [...] el hombre no los sabe utilizar se van agotar" (Carta). Consolidándose un JD3 que considera cómo la utilidad brindada por la naturaleza está regulada por un criterio ético, una postura hacia el uso correcto de los animales. Esta posición se reitera cuando considera las problemáticas para el manejo de los cultivos de yuca debido a la irrupción de la Danta, emergiendo así el criterio ético-emocional con referencia a la sensibilidad que le suscita la muerte de la Danta.

\section{Criterio Espiritual y jerarquías asociadas}

Para Juan la naturaleza tiene espíritu. Esta característica particular aludida a la naturaleza por él, permite configurar esta creencia como un criterio Espiritual, dado que no es tangible, físico, visible o definible con elementos de su entorno. En este sentido, pese a que Juan no encuentra cómo definir el espíritu de la naturaleza, éste se hace presente en sus creencias y está fuertemente arraigado en su discurso: "Investigador: ¿Crees qué la naturaleza tiene espíritu?- Juan: Sí. - Investigador: ¿Y cómo serían los espíritus de los animales o de la naturaleza o de las plantas?- Juan: No sé. [...] me han dicho pero no le he puesto bien cuidado a lo que me han dicho" (Entrevista).

De esta manera se consolida un JD1, ya que se hace manifiesto el hecho de que la naturaleza tiene espíritu, creencia fuertemente arraigada a lass tradiciones indígenas, un Ethos Sikuani; es de resaltar, que por la cercanía familiar de Juan a grupos Sikuani, su idea de naturaleza está mediada por un conocimiento del mundo natural que da paso a la consideración de "Madre Naturaleza". De este modo, Juan establece una relación entre el mundo natural, seres superiores y el Hombre, en la que se respeta, se quiere, y se cuida a la naturaleza como una madre. Lo anterior evidencia la relación entre el criterio Espiritual y el criterio E-E-E, configurando JD1.

Juan comenta que los seres que más conocen de la naturaleza son los animales, lo cual está en relación inmediata con su dibujo, a diferencia de sus compañeros, que se remiten directamente a las personas: "Investigador: ¿Tú quién crees qué sabe más sobre la naturaleza?- Juan: Los animales. [...] Porque ellos mantienen, mejor dicho, todo el mundo, ellos saben, saben de la naturaleza arto". (Entrevista). Sin embargo, al indagar sobre las personas que conocen sobre la naturaleza, se hace presente la escuela, por medio de sus maestros: "Investigador: ¿Y qué persona sabe más de la naturaleza que tú conozcas- Juan: De la naturaleza, los profesores. [...] Porque ellos son los que le enseñan a uno acerca de la naturaleza" (Entrevista).

\section{Conclusiones}

La presente comunicación estableció una forma de estudiar las ideas de naturaleza desde la clase de ciencias naturales, en la que se hacen presentes saberes tradicionales y escolares, cosmovisiones y el Ethos Sikuani. Se evidencio que la idea de naturaleza de Juan expresa cómo el contexto escolar tiene importancia en sus explicaciones; sin embargo, tienen más sentido y son más relevantes las explicaciones dadas en su cultura, ello se determinó por medio de los conglomerados de relevancias. Es de anotar que a pesar de que Juan se encuentre en un espacio escolar, tiene gran fuerza y alcance el Ethos Sikuani, en consecuencia, la cultura es el lugar de sentido de la idea de naturaleza de Juan.

El criterio espiritual orienta una serie de actuaciones, prácticas, acciones, emociones, posiciones y pensamientos particulares, que se vinculan de modo directo e indirecto con los demás. La naturaleza, para Juan, provee de bienestar al ser humano, ya que le brinda el alimento para su subsistencia; no obstante, al aparecer el criterio de utilidad ligado al criterio E-E-E, el discurso vislumbra una posición de cuidado hacia la naturaleza, en la cual están articuladas consideraciones de respeto, responsabilidad y regulación para con ella, al considerarla como fuente de vida, belleza y bienestar. 


\section{Agradecimientos}

A los niños del colegio Silvinio Caro Heredia; al cuerpo docente, en especial a las profesoras Claudia Pineda y Blanca Ballesteros. A la Doctora Adela Molina; a Diana Bautista y Viviana Zorro. Al Centro de Investigaciones y Desarrollo Científico de la Universidad Distrital (CIDC).

\section{Referencias}

Aikenhead, G. S. (1996). Science education: Border crossing into the subculture of science. Studies in Science Education, 27, 1-52.

Aikenhead, G. S. (1997). Toward a First Nations cross-cultural science and technology curriculum. Science y Education, 81, 217-238.

Aikenhead, G. S. (2001a). Integrating Western And Aboriginal Sciences: Cross-Cultural Science Teaching. Research in Science Education, 31 (3), 337-355.

Aikenhead, G. S. (2001b). Rekindling Traditions: Cross-Cultural Science y Technology Units Indigenous Knowledge Conference. Project. Obtenido desde, http://capes.usask.ca/ccstu

Aikenhead, G. S. y Jegede, O. J. (1999). Cross-cultural science education: A cognitive explanation of a cultural phenomenon. Journal of Research in Science Teaching, 36, 269-287.

Aikenhead, G. S. y Huntley, B. (1999). Teachers' views on Aboriginal students learning western and Aboriginal science. Canadian Journal for Native Education, 23, 159-175.

Aikenhead, G. S. y Olugbemiro, J. J. (1999). Cross-Cultural Science Education: A Cognitive Explanation of a Cultural Phenomenon. Journal of Research in Science Teaching, 36 (3), 269-287.

Candau, V. M. y Nehme, S. (2006, Mayo-Agosto). Conversas com... Sobre a didática e a perspectiva multi/intercultural. Educ. Soc., Campinas, 27, n (95), 471-493.

Cobern, B. y Aikenhead, G. S. (1997). Cultural Aspects of Learning Science. Association for Research in Science Teaching. Obtenido desde http://www.wmich.edu/slcsp/SLCSP121/culturalaspectsoflearningscience.pdf

Cobern, W., y Loving, C. (2001). Defining 'Science in a Multicultural World: Implications for Science Education. Science y Education, 85, 50-67.

Cobern, W. y Loving, C. (2000). Scientific Worldviews: A Case Study Of Four High School Science Teachers. Electronic Journal of Science Education, 5(2). Obtenido desde, http://wolfweb.unr. edu/homepage/crowther/ejse/cobernetal.html

Cobern, W. (1996a). Constructivism And Non-Western Science Education Research. International Journal of Science Education, 4(3), 287-302

Cobern, W. (1996b). World View, theory and conceptual change in science education. Science Education International, 80(5), $579-610$

Cobern, W. (1994a- Enero). Alternative Constructions Of Science And Science Education. Southern Africa Association for
Mathematics and Science Education Research, University of Durban-Westville, Durban, South Africa, 27-30.

Cobern, W. (1994b). World View, culture, and science education. Science Education International, 5(4), 5-8.

Cobern, W. (1991). World View Theory and Science Education Research. Manhattan-Kansas: NARST.

Jegede, O. J. y Ogawa, M. (1999, April). A study of science teachers' views about the connections between modern science and indigenous culture. Documento presentado en la Annual Meeting ofthe National Association for Research in Science Teaching, Boston.

Jegede, O. J. (1995). Collateral learning and the eco-cultural paradigm in science and mathematics education in Africa.Studies in Science Education, 25, p 97-137.

Jegede, O. J. y Okebukola, P. A. (1991). The effect of instruction on socio-cultural beliefs hindering. Journal of Research in Science Teaching, 28 (3), 275-285.

June, G. (1999). World View Analysis of Knowledge in a Rural Village: Implications for Science Education. Science Education, 83 (1), 77-95.

Kawasaki, K. (1997). An aspect of science education in Japan within the framework based on structural linguistics. In Ogawa, M. (Ed.). Effects of traditional cosmology on science education, 48-62.

Kawasaki, K. (1996). The concepts of science in Japanese and Western education. ScienceyEducation, 5, 1-20.

Molina, A. (2008). Contribuciones metodológicas para el estudio de relaciones entre contexto cultural e ideas sobre la naturaleza de niños y niñas. Bogotá: Universidad Distrital Francisco José de Caldas. Cuadernos Número 4.

Molina, A. (2007a). Analogía, pensamiento científico infantil y revalorización de las teologías y el antropomorfismo. TEA, Número extraordinario, 88-107.

Molina, A. (2007b, Julio-Diciembre). Relaciones entre contexto cultural y explicaciones infantiles acerca del fenómeno de las adaptaciones vegetales. Nodos y nudos, 3(23), 3-19.

Molina, A., Mojica, L. y López, D. (2006). Ideas de los niños y niñas sobre la naturaleza: estudio comparado. Revista Científica, 7, 41-62.

Molina. A. y Mojica, L. (2005). Presentación. En Enfoques Culturales en la Educación en Ciencias. Caso de la Evolución de la Vida, 7-8.

Molina, A. (2004). Investigaciones acerca de la enseñanza, el aprendizaje y los textos escolares en la evolución de la vida: enfoques culturales. Enfoques Culturales en la Educación en Ciencias. Caso de la Evolución de la Vida, 9-33.

Molina, A. y Mojica, L. (2004). Las teorías de la evolución en los textos escolares: análisis crítico histórico-epistemológico de Philipp Mathy. Enfoques Culturales en la Educación en Ciencias. Caso de la Evolución de la Vida, 69-91.

Molina, A. (2002). Conglomerado de Relevancias y formación científica de niños, niñas y jóvenes. Revista Científica (4), 187 200.

Molina, A. (2000). Conhecimento, Cultura e Escola: Um estudo de suas Inter-relações a partir das idéias dos alunos (8-12 anos) 
sobre os espinhos dos cactos. [Tesis Doctoral], Doutor em Educacao, Faculdade de Educação. Brasil: Universidade de São Paulo.

Moura De Macedo, R. C. (2007). Imagens E Narrativas Nos/Dos Murais: Dialogando Com Os Sujeitos Da Escola. Educ. Soc., Campinas, 28, 111-128.

Ogawa, M. (2000). International joint research on culture, language and gender sensitive science teacher education program. [Manuscrito no publicado]. Hiroshima University.

Ogawa, M. (1995). Science education in a multi-science perspective. Science y Education, 79, 583-593.

Ogawa, M. (1989a). An introductory Study on traditional Views of nature. The context of Pursuing Rationale for Science Education. Abstract.

Ogawa, M. (1989b). Beyond the tacit framework of "science" and "science education" among science educators. International Journal of Science Education, 11, 247-250.
Ogawa, M. (1986). Toward a New Rationale os science Education in a Nonwestern Society. European Journal of Science Educaction, 8, 113-119.

Okhee, L. (2003). Equity for linguistically and culturally diverse students. Science Education: A Research Agenda Teachers College Record. 105(3), 465-489.

Okhee, L. (1999). Equity Implications Based on the Conceptions of Science Achievement in Major Reform Documents. Review of Educational Research, 69(1), 83-115.

Riggs, E. M. (2004). Components Of Geoscience Education For Native American Communities Scence Education. Science Education, 89 (2), 296-313.

Shu-Chiu, L. (2005). "Models of The Heavens and the Earth: An Investigation of German and Taiwanese Students Alternative Conceptions of the Universe". International Journal of Science and Mathematics Education, 3, 295-325.

\section{Diálogo del conocimiento}

Recorrer las escuelas del país compartiendo y aprendiendo con los maestros colegas que se enfrentan a diario a una realidad que no es para nada halagüeña, me ha permitido reconocer una serie de dificultades que se evidencian cotidianamente en las escuelas y que particularmente, muy a pesar de las características contextuales tan marcadas y diversas entre las diferentes regiones, éstas problemáticas son casi generalizadas en el territorio nacional (por no hablar incluso de la realidad en otros países latinoamericanos) y en mi concepto muchas de ellas están asociadas con el "menosprecio" por los niños de la básica, y más aún por aquellos de las escuelas rurales y/o de "contextos no convencionales" (entiéndase por aquellas que no están en los cascos urbanos), desconociendo absolutamente la gran posibilidad que estos tienen de comprender del mundo natural, dado que para enfrentar su cotidianidad han aprendido a ser creativos, hábiles para observar, se cuestionan permanentemente, están despojados de prejuicios y no le temen a la experimentación.

Por lo anterior, encontrarme con un texto como el escrito por el profesor Andrés Venegas, que evidencia un acercamiento entre el mundo de la academia y la escuela real, me reconforta ya que logra establecer esos puentes necesarios de diálogo y construcción colectiva entre los investigadores universitarios y los maestros de las escuelas.

No haré puntualmente referencia al contenido del escrito porque él mismo se presenta de manera precisa y contundente, solo espero lograr hacer énfasis en la importancia que este tipo de trabajos de investigación rigurosa tiene para el desarrollo de la educación, no solo en ámbito nacional, en tanto se constituyen en puntos de referencia para los procesos de transformación de la escuela, arrojando elementos conceptuales y metodológicos que sin duda aportarán en el mejoramiento de las prácticas de los maestros, la formulación de proyectos de innovación articulados y contextualmente pertinentes, que propendan por el diseño e implementación de procesos de construcción de conocimiento y que posibiliten el repensamiento de las ideas fundamentales de las disciplinas desde una perspectiva cultural que trascienda la idea transmisionista eurocéntrica y anglosajona.

Siendo lo anterior una necesidad apremiante de abordar, hoy por hoy se reconocen diversas prácticas investigativas, que como la del profesor Venegas le dan sentido a la ciencia en la escuela, promueven la construcción de conocimiento de modo alternativo y contribuyen a la consolidación de un constructo pedagógico, epistemológico, didáctico y disciplinar más solido, en la medida en que se conoce con mayor cercanía y desde escenarios reales, cómo piensan y construyen los niños el conocimiento. 